Research Article

$\mathrm{J}$ Exp Clin Med

2021; 38(4): 461-465

doi: 10.52142 /omujecm.38.4.11

\title{
The efficacy of the boric acid-based maintenance therapy in preventing recurrent vulvovaginal candidiasis
}

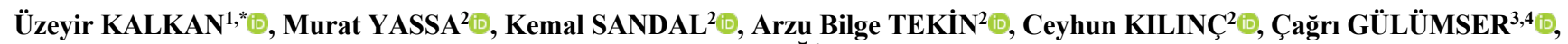 \\ Niyazi TUĞ $\breve{2}^{2}$ \\ ${ }^{1}$ Department of Obstetrics and Gynecology, Koç University Hospital, Istanbul, Turkey \\ ${ }^{2}$ Department of Obstetrics and Gynecology, Sehit Prof. Dr. Ilhan Varank Sancaktepe Training and Research Hospital, Istanbul, Turkey \\ ${ }^{3}$ Department of Obstetrics and Gynecology, School of Medicine, Yüksek Ihtisas University, Ankara, Turkey \\ ${ }^{4}$ Department of Public Health- Epidemiology, School of Medicine, Yüksek Ihtisas University, Ankara, Turkey
}

\begin{abstract}
Received: 04.03.2021 • Accepted/Published Online: 24.03.2021 • Final Version: 30.08 .2021
Abstract

Current gold-standard treatment of recurrent vulvovaginal candidiasis (RVVC) is mainly based on maintenance with fluconazole. Moderate to high recurrence rates at long-term use and secondary fluconazole resistance emerge as reasons to seek for new topical maintenance regimens. In this study, it is aimed to assess the efficacy and safety of boric acid-based treatment approach to treat clinical RVVC. In this retrospective study, patients who were diagnosed with RVVC received a treatment package for six months that consist of induction with boric acid vaginal suppositories $600 \mathrm{mg}$ daily for 14 nights followed by maintenance for five nights starting with every fifth day of the menstrual cycles; a vaginal estriol-lactobacilli combination; and several rigorous life-style changes. The success was defined as the absence of symptomatic recurrence during the follow-up. Success rate at the first year was found to be $94.8 \%$ in a total of 173 patients. Mild, reversible side effects were observed in five patients $(2.9 \%)$. Boric acid, along with a vaginal estriol-lactobacilli combination and lifestyle changes can be a safe and effective alternative in lieu of potent systemic antifungal drugs as a first-line treatment for the patients referred with RVVC.
\end{abstract}

Keywords: boric acid, lifestyle changes, recurrent vulvovaginal candidiasis, vaginal flora

\section{Introduction}

Recurrent vulvovaginal candidiasis (RVVC) is a challenging morbidity both for patients and clinicians. It substantially impairs the quality of life and sexual function due to a variety of symptoms that include vaginal discharge, vulvar itch, soreness, and dyspareunia (1).

Global annual prevalence of RVVC was estimated as 3871 per 100,000 patients and the 25-34-year age group had the highest prevalence with $9 \%$ (2). RVVC is very frequent that almost one-tenth of patients report four or more episodes in their lifetime. The probability of multiple recurrences was found to be $10 \%$ for patients aged 25 years and increased to $25 \%$ at the age of 50 years (3). Almost $5 \%$ of patients of reproductive age after an initial episode of VVC will progress to recurrent disease (4).

Candida albicans is also responsible for most infections in patients with RVVC as well as acute sporadic VVCs. Of the non-albicans Candida species, Candida glabrata is the most frequently isolated species from the vagina in symptomatic and asymptomatic patients (5).

The cause of RVVC is thought to be multifactorial and the mechanism behind the resistance to antifungal medications is not well known (6). Genetic susceptibility reduced mannose- binding lectin and host exaggerated inflammatory response are suggested theories of recurrence, however, a tool to control the host mucosal reaction still does not exist (5). The current therapeutical approach mainly aims to reduce and subsequently suppress the vaginal fungal load with several regimens, particularly with azoles, but not all Candida strains are azole-sensitive such as Candida glabrata.

Current gold-standard treatment of RVVC includes maintenance with fluconazole once weekly regimens that often needs to be continued for several years. The recurrence rate at one year with fluconazole following the maintenance regimen varies between $57-77 \%(7,8)$. On the other hand, widespread use of fluconazole for either acute sporadic or recurrent cases caused an emerging trend of secondary fluconazole resistance (9).

The initial drug of choice in cases of azole-resistant nonalbicans Candida species is vaginal boric acid $600 \mathrm{mg}$ daily for 14 days (10). Vaginal boric acid was proposed to be effective with rapid relief of symptoms and culture negativity after two weeks of use in treating RVVC and further combination with other regimens were suggested where it alone fails (5, 11-13). 
Considering the pharmaceutical and economic burden of the long-term use of fluconazole and the success of boric acid in azole-resistant cases, we hypothesized that boric acid may be a successful tool to be used primarily in treating RVVC. It was aimed to assess the efficacy and safety of boric acidbased treatment approach including a package of behavioral changes to treat clinical RVVC in a pragmatic trial.

\section{Materials and methods}

The study presented retrospective analysis of prospectively collected data yielded at a tertiary health care centre from June 2017, through July 2019.

Recurrent VVC was defined as four or more symptomatic episodes over a 12-month period that shows resolution between the episodes. Progress with the antifungal treatment and exacerbation with the use of systemic antibiotics are also agreed to be relevant to RVVC. At least one of those episodes was confirmed by microscopy with the presence of blastospores, pseudohyphae and neutrophils during cytology. Patients included to the trial were aged over 18 years to 50 years old, not in menopause, did not have systemic illnesses and diagnosed with RVVC. Patients who had any other sexually transmitted disease, malignity, gynaecological anatomic disorder, any morbidity known to cause susceptibility to candidiasis including diabetes mellitus, pregnancy, patients currently on any related medications such as antibiotics or corticosteroids and who had used antifungal medication in the week before entry were excluded prior to the treatment. The study was conducted in according to the Helsinki Declaration, 2008 (http://www.wma.net/en/30publications/10policies/b3/index.h tml). The local institutional administration board approved the study (No:2020/42). Preliminary results have been presented in $17^{\text {th }}$ National Gynecology and Obstetrics Congress, Antalya, Turkey.

All patients underwent a detailed urogynecological examination by the first author. Patients with mixed infection, urinary incontinence or symptomatic female genital prolapse over POP-Q grade II were excluded after the enrollment and received proper treatment. As discussed later, authors had the belief that urogynecological problems may substantially disturb the vulvovaginal flora.

Patients who met the entry criteria received a treatment package for six months that consist of three main components (Fig. 1):

(1) Induction therapy was provided with boric acid vaginal suppositories $600 \mathrm{mg}$ daily for 14 nights followed by maintenance for five nights starting with every fifth day of the menstrual cycles considering that menstrual bleeding should significantly decrease or stop by that time. If mucosal irritation occurs, the dose was reduced to $300 \mathrm{mg}$ daily.

(2) A vaginal estriol-lactobacilli combination (Gynoflor ${ }^{\circledR}$, Abdi Ibrahim Ilac Pazarlama A.S., Medinova
Ltd., Switzerland) following five-day boric acid administration to treat disrupted vaginal microflora that contains at least $1 \times 108$ colony-forming units of live Lactobacillus acidophilus, $0.03 \mathrm{mg}$ estriol and approximately $600 \mathrm{mg}$ lactose. This regimen was recommended to be repeated if patient had used any antibiotics for any reason after she was enrolled to the study.

(3) Several rigorous lifestyle and behavioral changes are seen. Couple was recommended to wash their external genitalia with warm water and mild or unscented soap prior to coitus. Patients was strained from vaginal washes, wipes, and douche with water and/ or soap, local irritants, oral sex and use of vaginal objects including tampon or vibrators. Patients was recommended to wear baggily and cotton clothes, to consume more yoghurt and kefir, reduce or quit smoking. Patients was suggested to avoid vaginal sex until their symptoms improved.

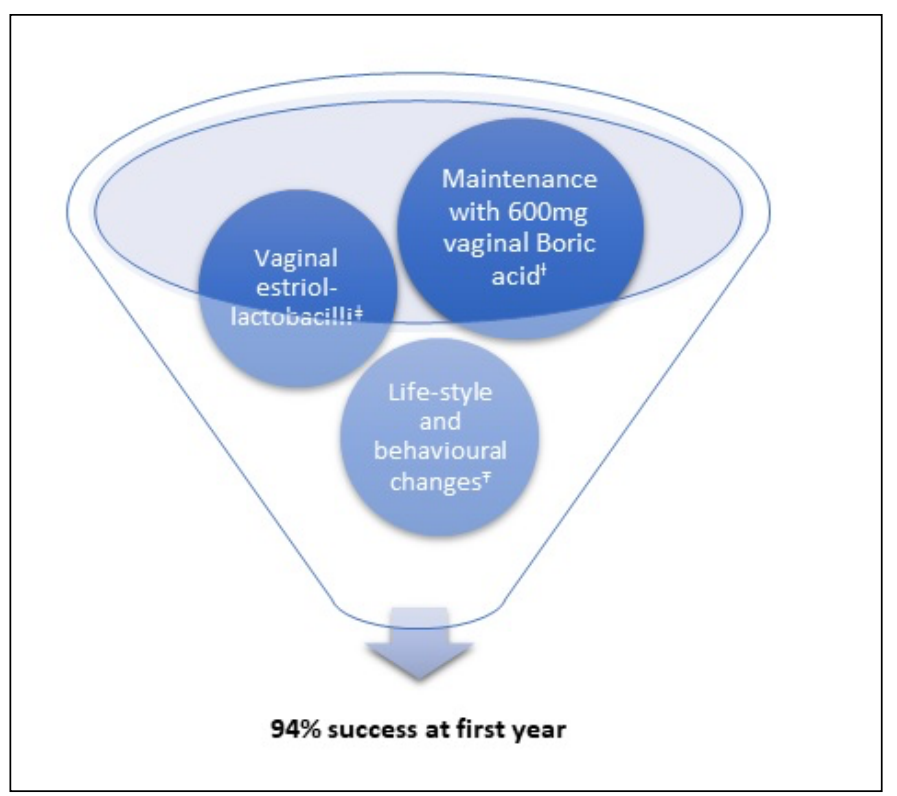

Fig. 1. Boric acid-based combination therapy for RCCV

Patients were required to return to the gynaecological outpatient clinic at their first, third and sixth months and the first year of the boric acid-based maintenance therapy for RVVC. The success was defined as the absence of symptomatic recurrence as stated by the patient during the follow-up. If recurrence occurs, fluconazole $150 \mathrm{mg}$ orally every 72 hours in a total of three doses was suggested to ensure clinical remission. When oral therapy needs to be avoided $600 \mathrm{mg}$ of intravaginal fluconazole administered on day one and four as an alternative. Therapy outcome was assessed with descriptive statistics.

\section{Results}

A total of 221 patients with RVVC have included. Seventeen and twenty-five patients were excluded before and the after the enrollment, respectively. Before the enrollment, nine patients due to recent use of different or unknown antifungal medication, six for premalignant cervical lesion and two for uncontrolled diabetes mellitus were excluded. After the 
enrollment, patients with significant urogynecological symptoms were excluded and scheduled to midurethral mini sling in 13 patients, laparoscopic lateral suspension with mesh in five, anterior and posterior colporrhaphy in three, transobturator tape in two and combination of those in two patients. Six patients were lost to follow-up. In the end, a total of 173 patients were included to the descriptive analysis.

The mean age of the patients was $31.5 \pm 4.4$ years $(\mathrm{Min}=$ 24 , $\max =48$ ). The median parity was two with an interquartile range of one $(\operatorname{Min}=0, \max =5)$.

Induction therapy was successful in all cases. Overall success rate at the first year was found to be $94.8 \%$ out of 173 patients that was treated with boric acid-based maintenance therapy for RVVC.

Out of nine patients who had recurrence VVC episodes, one was at her first month, two were at third month, two were at sixth month, one was at ninth month and three were at her $12^{\text {th }}$ month of the treatment (Fig. 2). Patient who had the earliest recurrence at the first month during the maintenance therapy was observed to have intrauterine contraception device. Five of the recurrent cases $(55.5 \%)$ were observed during the maintenance therapy and the rest $(44.5 \%)$ was observed after the maintenance therapy that lasted for six months.

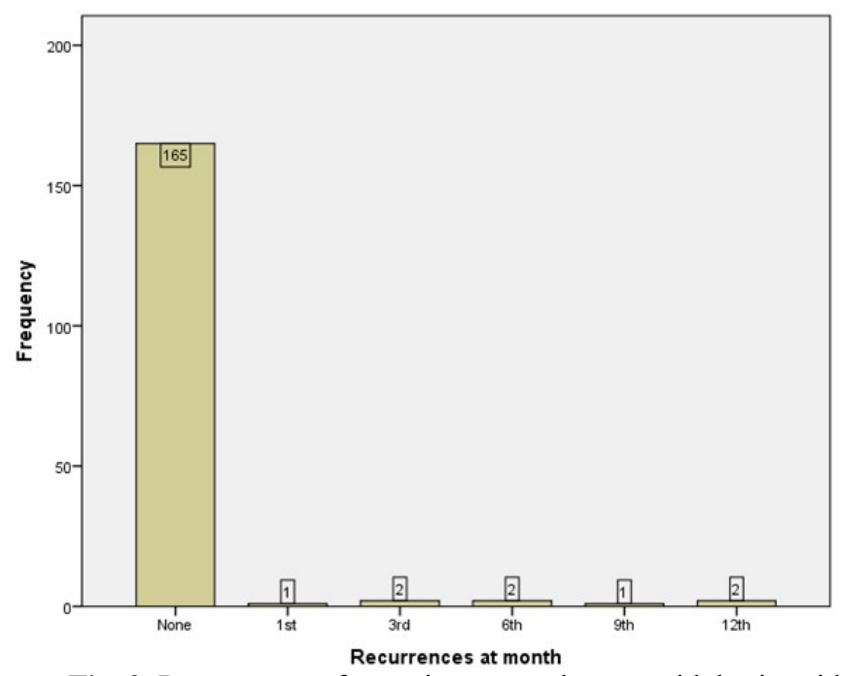

Fig. 2. Recurrences after maintenance therapy with boric acid

Mild or moderate side effects were observed in five patients $(2.9 \%)$. Local burning sensation and erythema were observed in four and one of patients, respectively. Only the patient with erythema quitted the boric acid-based maintenance treatment and changed to fluconazole $150 \mathrm{mg}$ orally once a week for six months. She was recurrence free at the at first year follow-up. The boric acid dose was reduced to $300 \mathrm{mg}$ daily in the four patients with mild local burning sensation and none of them had recurrence.

\section{Discussion}

The findings of this study may promote the clinical use of boric acid in lieu of potent antifungal drugs as a first-line treatment for the patients referred with RVVC. The overall success rate was almost $95 \%$ at the first year with six months use of boric acid-based approach including supporting the vaginal microflora and the lifestyle and behavior changes. The side effect of the regimen was observed to be mild and low with $3 \%$ rate.

The current most recommended treatment of RVVC is known as suboptimal. The most accepted regimen to treat azole sensitive RVVC is the suppression with fluconazole, however, it is thought that almost half of the patients will have another recurrence episode within 3-4 months following the cessation of fluconazole (5). In a recent review of the current guideline recommendations, the regimens suggested by the most guidelines were found to be particularly ineffective with only $43 \%$ of patients were disease-free at the first year (1). By the increased secondary fluconazole resistance due to its overuse, the need of new approaches was proclaimed considering management of fluconazole refractory vulvovaginitis was reported to be difficult $(9,14)$. In addition, new Candida agents were shown to be emerging that is also resistant to azoles (15).

The optimal duration of suppressive therapy with azoles is currently unknown (4). Long-term treatment with systematic agents was suggested to be given following in vitro susceptibility tests and therefore, boric acid may have a role in long-term maintenance treatment where the azole susceptibility is unknown (9).

Despite the lack of published data in the literature, it is a known fact that clinicians worldwide use long-term maintenance with intravaginal boric acid in the treatment of RVVC with high patient satisfaction and few adverse events (16). In a systematic review; boric acid step forward as an economic and safe alternative to azoles for the ordinary treatment of VVC with the advantage of not inducing drug resistance and of not discriminating between the Candida species (12). Boric acid has also shown to have dosedependent antimicrobial activity against Trichomonas vaginalis (17).

RVVC is caused by host factors rather than infecting with a more virulent strain or reintroduction of the pathogen to the vulvovaginal tract (4). Host factors include persistence of Candida and the disturbance of vaginal microflora. Currently, boric acid is not used as a first-line treatment, mainly because less known about its mechanism of action. At this point, it is recently showed by Schmidt et. al. that boric acid may restore the persistence by inhibiting the several metabolic pathways including glycolysis, fermentation, and the mitochondrial activity (18). Boric acid was also shown to induce cell autofluorescence, decrease catalase activity and the cell size, and subsequently promote the programmed cell death (19).

The regimen used in the current study includes an intermittent maintenance therapy with monthly boric acid for 
five nights starting with every fifth day of the menstrual cycles. This step aimed to keep the vaginal fungal load at reduced levels. As discussed by Sobel, there is currently no other method to control the host mucosal reaction but facilitating yeast antigen tolerance with decreased viral load (5). Guaschino et al. examined the efficacy of long-term use of vaginal boric acid in the cure and prevention of recurrent vulvovaginal candidiasis (20). They used a similar concept of maintenance regimen to the current study but not the same in dosage and the timing. Monthly maintenance therapy has given daily for five days from the first day of the menstrual cycle in contrary to our postmenstrual approach. We postulate that menstrual approach can seriously impair the patient compliance and variances in bleeding patterns can substantially decrease the effect of vaginal boric acid. In a similar manner, Reichman et. al. delayed the vaginal use of boric acid during the menses in their study assessing a similar regimen in treating bacterial vaginosis (21). Nevertheless, it should be noted that this is an empiric regimen that needs to be validated by further prospective trials.

Secondly, a vaginal estriol-lactobacilli combination was added following the treatment with boric acid. Lactobacilli are essential in maintaining the vaginal flora by preventing the colonization of pathogens (22). A recent systematic review that investigating the impact of vaginal probiotics on VVC cure has reached two important conclusions (23). Firstly lactobacilli-containing vaginal probiotics were found to be encouraging in cure and prevention of bacterial vaginosis, however, the benefits for VVC were not promotive. Secondly, the probiotic strains did not persist after cessation of the treatment that suggests probiotics did not colonize the vagina. At this stage, the lifestyle and the behavioral changes become prominent that provide the persistence of reduced viral load and decrease the triggering stimuli, as in the current study.

The safety of the vaginal use of boric acid was well documented (12). The adverse outcomes following the vaginal boric acid were observed to be low in this study with only mild and reversible side effects. To be noted, watery discharge was not considered as a side effect and has told to the patients during the counselling that this can often be seen. Expectedly, there was no complain or cessation of the treatment due to watery discharge that is common in the morning.

The high success of this study can be explained by several aspects. The rigorous lifestyle recommendations and the vaginal flora support should have enhanced the results. To the best of our knowledge, this is the first study that has performed a detailed urogynecological examination and excludes patients accordingly among the studies investigating the treatment of RVVC. Contrary to common popular belief, it is now known that a urinary microbiome exists (24). The urine may act as a reservoir for pathogens and cause recurrences for infections such as bacterial vaginosis (25). We also speculate that urogynecological problems including urinary incontinence and pelvic organ prolapse may disturb the vulvovaginal flora due to chronic irritation of urine or persistent exposure to the external environment.

The main limitation of this study was that the diagnosis of RVVC was not supported by culture in this study due to technical paucity. The absence of the control group to compare with the success of boric acid was another limitation. A further prospective case-control study is needed. There is also a causative bias that the success may not be solely linked to the use of boric acid. On the other hand, this study encompasses a realistic population and a vast number of patients with RVVC. The data derived from the current study can fill a gap in the lacking evidence for the efficacy, safety, and the need for long-term maintenance regimen with boric acid.

The overall success rate of boric acid-based approach reached to $95 \%$ at a one-year follow-up. Boric acid, along with a vaginal estriol-lactobacilli combination and lifestyle changes can be a safe and effective alternative in lieu of potent systemic antifungal drugs as a first-line treatment for the patients referred with RVVC.

\section{Conflict of interest}

The authors report no conflict of interest.

\section{Funding}

None.

\section{Acknowledgement}

None.

\section{References}

1. Matheson A, Mazza D. Recurrent vulvovaginal candidiasis: A review of guideline recommendations. Aust $\mathrm{N} \mathrm{Z} \mathrm{J} \mathrm{Obstet}$ Gynaecol. 2017; 57(2):139-45. doi: 10.1111/ajo. 12592.

2. Denning DW, Kneale M, Sobel JD, Rautemaa-Richardson R. Global burden of recurrent vulvovaginal candidiasis: a systematic review. Lancet Infect Dis. 2018; 18(11): e339-e347. doi: 10.1016/S1473-3099(18)30103-8.

3. Foxman B, Muraglia R, Dietz JP, Sobel JD, Wagner J. Prevalence of recurrent vulvovaginal candidiasis in 5 European countries and the United States: results from an internet panel survey. J Low Genit Tract Dis. 2013;17(3):340-5. doi: 10.1097/LGT.0b013e318273e8cf.

4. Rao VL, Mahmood T. Vaginal discharge. Obstetrics, Gynaecology \& Reproductive Medicine. 2020; 30 (1): 11-8. doi.org/10.1016/j.ogrm.2019.10.004.

5. Sobel JD. Recurrent vulvovaginal candidiasis. Am J Obstet Gynecol. 2016; 214(1):15-21. doi: 10.1016/j.ajog.2015.06.067.

6. Ramírez-Lozada T, Espinosa-Hernández VM, Frías-De-León MG, Martínez-Herrera E. Update of Vulvovaginal Candidiasis in Pregnant and Non-pregnant Patients. Curr Fungal Infect Rep. 2019; 13(4):181-90. doi.org/10.1007/s12281-019-00357-3.

7. Donders G, Bellen G, Byttebier G, Verguts L, Hinoul P, Walckiers $R$, et al. Individualized decreasing-dose maintenance fluconazole regimen for recurrent vulvovaginal candidiasis (ReCiDiF trial). Am J Obstet Gynecol. 2008; 199(6): 613.e1-9. doi: 10.1016/j.ajog.2008.06.029. 
8. Sobel JD, Wiesenfeld HC, Martens M, Danna P, Hooton TM, Rompalo A, et al. Maintenance fluconazole therapy for recurrent vulvovaginal candidiasis. N Engl J Med. 2004; 351(9):876-83. doi: 10.1056/NEJMoa033114.

9. Marchaim D, Lemanek L, Bheemreddy S, Kaye KS, Sobel JD. Fluconazole-resistant Candida albicans vulvovaginitis. Obstet Gynecol. 2012;120(6):1407-14. doi: 10.1097/aog.0b013e31827307b2.

10. Sobel JD, Sobel R. Current treatment options for vulvovaginal candidiasis caused by azole-resistant Candida species. Expert Opin Pharmacother. 2018; 19(9):971-7. doi: $10.1080 / 14656566.2018 .1476490$.

11. Sobel JD, Chaim W, Nagappan V, Leaman D. Treatment of vaginitis caused by Candida glabrata: use of topical boric acid and flucytosine. Am J Obstet Gynecol. 2003 Nov;189(5):1297300. doi: 10.1067/s0002-9378(03)00726-9.

12. Iavazzo C, Gkegkes ID, Zarkada IM, Falagas ME. Boric acid for recurrent vulvovaginal candidiasis: the clinical evidence. J Womens Health (Larchmt). 2011; 20(8):1245-55. doi: 10.1089/jwh.2010.2708.

13. De Seta F, Schmidt M, Vu B, Essmann M, Larsen B. De Seta F, et al. Antifungal mechanisms supporting boric acid therapy of Candida vaginitis. J Antimicrob Chemother. 2009; 63(2): 32536. doi: $10.1093 / \mathrm{jac} / \mathrm{dkn} 486$.

14. Córdoba S, Taverna C, Vivot W, Szusz W, Vivot M, Isla G, et al. Emergence of Resistance to Fluconazole in Candida albicans Isolated from Vaginal Discharge. Curr Fungal Infect Rep. 2018; (12): 155-60. doi.org/10.1007/s12281-018-0329-6.

15. Aznar-Marin P, Galan-Sanchez F, Marin-Casanova $P$, GarcíaMartos P, Rodríguez-Iglesias M. Candida nivariensis as a New Emergent Agent of Vulvovaginal Candidiasis: Description of Cases and Review of Published Studies. Mycopathologia. 2016; 181(5-6): 445-9. doi: 10.1007/s1 1046-015-9978-y.

16. Powell A, Ghanem KG, Rogers L, Zinalabedini A, Brotman RM, Zenilman J, et al. Clinicians' Use of Intravaginal Boric Acid Maintenance Therapy for Recurrent Vulvovaginal Candidiasis and Bacterial Vaginosis. Sex Transm Dis. 2019; 46(12):810-2. doi: 10.1097/OLQ.0000000000001063.
17. Thorley N, Ross J. Intravaginal boric acid: is it an alternative therapeutic option for vaginal trichomoniasis? Sex Transm Infect. 2018; 94(8): 574-7. doi: 10.1136/sextrans-2017-053343.

18. Schmidt M, Tran-Nguyen D, Chizek P. Influence of boric acid on energy metabolism and stress tolerance of Candida albicans. J Trace Elem Med Biol. 2018; 49:140-5. doi: 10.1016/j.jtemb.2018.05.011.

19. Beach T, Hart B, Larsen B. Stress response in Candida albicans induced by boric acid. Journal of Advances in Medicine and Medical Research. 2016; 15 (8): 1-11. doi: 10.9734/BJMMR/2016/25887.

20. Guaschino S, De Seta F, Sartore A, Ricci G, De Santo D, Piccoli $\mathrm{M}$, et al. Efficacy of maintenance therapy with topical boric acid in comparison with oral itraconazole in the treatment of recurrent vulvovaginal candidiasis. Am J Obstet Gynecol. 2001; 184(4): 598-602. doi: 10.1067/mob.2001.111938.

21. Reichman O, Akins R, Sobel JD. Boric acid addition to suppressive antimicrobial therapy for recurrent bacterial vaginosis. Sex Transm Dis. 2009; 36(11):732-4. doi: 10.1097/OLQ.0b013e3181b08456.

22. Ozkinay E, Terek MC, Yayci M, Kaiser R, Grob P, Tuncay G. The effectiveness of live lactobacilli in combination with low dose oestriol (Gynoflor) to restore the vaginal flora after treatment of vaginal infections. BJOG. 2005;112(2):234-40. doi: 10.1111/j.1471-0528.2004.00329. x.

23. van de Wijgert J, Verwijs MC. Lactobacilli-containing vaginal probiotics to cure or prevent bacterial or fungal vaginal dysbiosis: a systematic review and recommendations for future trial designs. BJOG. 2020; 127(2):287-99. doi: 10.1111/14710528.15870 .

24. Thomas-White K, Brady M, Wolfe AJ, Mueller ER. The bladder is not sterile: History and current discoveries on the urinary microbiome. Curr Bladder Dysfunct Rep. 2016;11(1):18-24. doi: 10.1007/s11884-016-0345-8.

25. Gottschick C, Deng ZL, Vital M, Masur C, Abels C, Pieper DH, et al. The urinary microbiota of men and women and its changes in women during bacterial vaginosis and antibiotic treatment. Microbiome. 2017; 5(1):99. doi: 10.1186/s40168-017-0305-3. 